\title{
Estudios físico-químicos de miscelas de aceites vegetales. XV. Aplicación de las ecuaciones de Andrade y Krone a disoluciones de miristato de metilo y ciclohexano, hexano, tetracloroetileno o tricloroetileno
}

\author{
Por Ma J. Muñoz Cueto, M. Galán Vallejo, M. Rodríguez Rodríguez y R. Martín Minchero \\ Departamento de Ingeniería Química de la Universidad de Cádiz. Apartado 40. 11510 Puerto Real (Cádiz)
}

RESUMEN

Estudios físico-químicos de miscelas de aceites vegetales. XV. Aplicación de las ecuaciones de Andrade y Krone a disoluciones de miristato de metilo y ciclohexano, hexano, tetracloroetileno o tricloroetileno.

Se aplican las ecuaciones de Andrade y Krone, que relacionan la viscosidad de los líquidos con la temperatura, a disoluciones de compuestos orgánicos de diferentes estructuras químicas formadas por miristato de metilo y ciclohexano, hexano, tetracloroetileno o tricloroetileno, en el intervalo de temperatura de 283 a $313 \mathrm{~K}$, comparándose los resultados obtenidos con los experimentales.

PALABRAS-CLAVE: Aceite vegetal - Disolvente orgánico Miristato de metilo - Miscela - Viscosidad.

\section{SUMMARY}

Physico-chemical studies concerning miscelles of vegetable oils. XV. Application of Andrade and Krone equations to methyl mirystate solutions in cyclohexane, hexane, tetrachloroethylene or trichloroethylene.

Andrade and Krone equations, relating viscosity of liquids and temperature, are applied to solutions of organic compounds with different chemical structures, constituted by methyl mirystate and ciclohexane, hexane, tetrachloroethylene or trichloroethylene. Temperature range studied is $283-313 \mathrm{~K}$. The results are compared with experimental data.

KEY-WORDS: Methyl myristate - Miscella - Organic solvent Vegetable oil - Viscosity.

\section{INTRODUCCIÓN}

Las teorías y/o ecuaciones desarrolladas sobre viscosidad de líquidos newtonianos se pueden clasificar en dos grupos: teorías basadas en propiedades moleculares (Andrade, 1934; Kirkwood, 1968; Mayer y Montroll, 1941; Mayer, 1947 y Born y Green, 1946) y teorías basadas en modelos. De estas últimas, hay que destacar las teorías basdas en el modelo de energía de activación (Ewell y Eyring, 1937; Ree y cols., 1964; Eyring e Ihon, 1969; Hogemboom, 1967 y Faeber y cols., 1970).

Relacionadas con el modelo de energía de activación se han desarrollado aproximaciones empíricas que han conducido a relaciones útiles para interpolar datos de viscosidad. La relación empírica más usada es la propuesta por Andrade, 1930:

$$
\eta=\lambda e^{E / R T}
$$

donde " $\eta$ " es el valor de la viscosidad dinámica (mPa. s) a la temperatura absoluta "T" (K), " $\lambda$ " $y$ " $E$ " son constantes características de cada líquido y " $\mathrm{R}$ " es la constante de los gases perfectos.

Según la ley de distribución de Maxwell-Boltzmann, el número de moléculas que poseen la energía necesaria

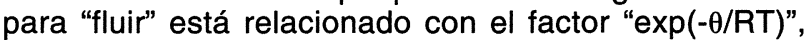
donde " $\theta$ " es la energía de activación molar de flujo. La velocidad de flujo, y por tanto la viscosidad, inversa de la fluidez, dependerá del mismo, como indica la ecuación (1) en la cual se puede tomar "E" como equivalente a la energía de activación de flujo.

El factor " $\lambda$ " de la ecuación (1) depende fundamentalmente del peso molecular y del volumen molar del líquido, de acuerdo con la definición de Van der Waals (Andrade, 1934).

Posteriormente (Krone, 1983), se ha propuesto otra ecuación que relaciona la viscosidad con la temperatura basada en la hipótesis de que el transporte de cantidad de movimiento tiene lugar a través de capas adyacentes de moléculas en movimiento relativo. Las moléculas que intervienen en el flujo permanecen en sus respectivas capas sometidas a la acción del campo de fuerzas de sus vecinas, al tiempo que interaccionan con moléculas de la capa adyacente. Por tanto, en el cálculo del transporte de cantidad de movimiento, debe incluirse no la masa de una molécula, sino la masa de las moléculas que intervienen en dicha interacción como una unidad. La ecuación propuesta es de la forma:

$$
\ln \left[\eta /\left(T-T_{0}\right)\right]=\left(E^{\# / R T}\right)+\ln \beta
$$

donde " $\eta$ " es la viscosidad dinámica, " $T_{0}$ " un parámetro cuyo producto por la constante de Boltzmann, representa la energía térmica media que no es aprovechada para que aumente la frecuencia de interacciones entre moléculas de capas adyacentes, " $E \# "$ la energía de activación necesaria para crear un hueco; " $R$ " la constante de los gases perfectos y " $\beta$ " un parámetro definido por la expresión:

$$
\beta=(\alpha \delta n k M) / h N
$$

siendo " $\alpha$ " la fracción de movimientos térmicos que producen la interacción entre capas adyacentes, " $\delta$ " la distancia entre capas medida de eje a eje, " $n$ " el número de moléculas por unidad de superficie, " $\mathrm{M}$ " el peso molecular, "N" el número de Avogadro, "k" la constante de Boltzmann y "h" la constante de Planck. 


\section{DISCUSIÓN}

\section{Ecuación de Andrade}

Se ha aplicado la ecuación (1) a los datos experimentales de viscosidad (Muñoz, 1990) modificada de la forma que se indica a continuación, tomando como temperatura de referencia del intervalo de medida $303 \mathrm{~K}$. De esta forma el parámetro "In $\eta_{303}$ " debe coincidir, para cada disolución, con el valor experimental del logaritmo neperiano de la viscosidad a $303 \mathrm{~K}$.

$$
\ln \eta=\ln \eta_{303}+E / R[(1 / T)-(1 / 303)]
$$

Los valores de los parámetros "In $\eta_{303}$ " y "E" se indican en la Tabla I. Los errores porcentuales máximos que les afectan, para una probabilidad del $95 \%$, son del $8 \%$ y $7 \%$, respectivamente. Los valores del porcentaje de la varianza no justificada por la regresión, [VNJ $=100\left(1-r^{2}\right)(I-1) /(I-2)$; donde "r" es el coeficiente de correlación lineal e "l" el número de pares de datos] son inferiores a 0,42.

\begin{tabular}{|c|c|c|c|c|c|c|c|c|}
\hline \multirow[b]{2}{*}{$X_{\text {ester }}$} & \multicolumn{2}{|c|}{$\begin{array}{c}\text { Miristato } \\
\text { de metilo } \\
\text { Ciclo- } \\
\text { hexano }\end{array}$} & \multicolumn{2}{|c|}{$\begin{array}{l}\text { Miristato } \\
\text { de metilo } \\
\text { Hexano }\end{array}$} & \multicolumn{2}{|c|}{$\begin{array}{c}\text { Miristato } \\
\text { de metilo } \\
\text { Tetracloro- } \\
\text { etileno }\end{array}$} & \multicolumn{2}{|c|}{$\begin{array}{l}\text { Miristato } \\
\text { de metilo } \\
\text { Tricloro- } \\
\text { etileno }\end{array}$} \\
\hline & $\begin{array}{c}E \\
\text { (cal/mol) }\end{array}$ & $\ln \eta_{303}$ & $\begin{array}{c}E \\
(\mathrm{cal} / \mathrm{mol})\end{array}$ & $\ln \eta_{303}$ & $\underset{(\mathrm{cal} / \mathrm{mol})}{E}$ & $\ln \eta_{303}$ & $\begin{array}{c}E \\
\text { (callmol) }\end{array}$ & $\ln _{\eta} \eta_{303}$ \\
\hline 0.10 & 2616 & 0.124 & 2396 & -0.715 & 2013 & 0.245 & 1895 & -0.226 \\
\hline 0.20 & 2824 & 0.298 & 2397 & -0.368 & 2472 & 0.349 & 2238 & 0.037 \\
\hline 0.30 & 3093 & 0.459 & 2481 & -0.107 & 2807 & 0.505 & 2669 & 0.304 \\
\hline 0.40 & 3469 & 0.641 & 2600 & 0.119 & 3133 & 0.659 & 3150 & 0.510 \\
\hline 0.50 & 3701 & 0.762 & 2943 & 0.317 & 3374 & 0.791 & 3492 & 0.694 \\
\hline 0.60 & 3939 & 0.889 & 3343 & 0.519 & 3674 & 0.917 & 3727 & 0.852 \\
\hline 0.70 & 4135 & 1.012 & 3563 & 0.731 & 3833 & 1.026 & 3904 & 0.987 \\
\hline 0.80 & 4195 & 1.120 & 3771 & 0.931 & 3983 & 1.129 & 3935 & 1.104 \\
\hline 0.90 & 4118 & 1.215 & 3942 & 1.123 & 4001 & 1.218 & 4086 & 1.210 \\
\hline
\end{tabular}

Los valores de los parámetros "In $\eta_{303}$ " y "E" varían de forma creciente con el contenido de éster metílico en la disolución, observándose para la misma fracción molar de éster metílico, un aumento del valor de " $E$ " en el sentido de las disoluciones con hexano, tricloroetileno o tetracloroetileno y ciclohexano.

Las diferencias existentes entre el valor experimental de la viscosidad de las disoluciones a $303 \mathrm{~K}$ y el valor de " $\eta_{303}$ " obtenido del ajuste de los resultados experimentales a la ecuación (3) son inferiores al $1 \%$ en todos los casos.

\section{Ecuación de Krone}

Al aplicar la ecuación (2) a los mismos valores experimentales de viscosidad, se ha encontrado que el valor de $T_{0}$ que hace mínimo el valor de la raiz cuadrada de las desviaciones cuadradas medias,

$\left[\operatorname{RMSD}(\eta)=\left[\Sigma\left(\eta_{\text {estimado }}-\eta_{\text {experimental }}\right)^{2 / I}\right]^{1 / 2}\right.$ es $0 \mathrm{~K}$.
Los valores de los parámetros "In $\beta$ " y " $E$ \#" obtenidos se indican en la Tabla II. Los errores porcentuales máximos que los afectan, para una probabilidad del $95 \%$, son del $3 \%$ y $5 \%$, respectivamente, siendo los valores del porcentaje de la varianza no justificada por la regresión inferiores a 0,28.

\begin{tabular}{|c|c|c|c|c|c|c|c|c|}
\hline \multirow[b]{2}{*}{ Xéster } & \multicolumn{2}{|c|}{$\begin{array}{c}\text { Miristato } \\
\text { de metilo } \\
\text { Ciclo- } \\
\text { hexano }\end{array}$} & \multicolumn{2}{|c|}{$\begin{array}{l}\text { Miristato } \\
\text { de metilo } \\
\text { Hexano }\end{array}$} & \multicolumn{2}{|c|}{$\begin{array}{c}\text { Miristato } \\
\text { de metilo } \\
\text { Tetracloro- } \\
\text { etileno }\end{array}$} & \multicolumn{2}{|c|}{$\begin{array}{c}\text { Miristato } \\
\text { de metilo } \\
\text { Tricloro- } \\
\text { etileno }\end{array}$} \\
\hline & $\begin{array}{c}\mathrm{E}^{*} \\
(\mathrm{cal} / \mathrm{mo}\end{array}$ & ln $\ln \beta$ & $\begin{array}{c}\mathrm{E}^{\prime} \\
(\mathrm{cal} / \mathrm{mol})\end{array}$ & 1) $\ln \beta$ & $\begin{array}{c}\mathrm{E}^{\prime} \\
\text { (cal/mol }\end{array}$ & In $\ln \beta$ & $\begin{array}{c}\mathrm{E}^{*} \\
\text { (cal/mo }\end{array}$ & ol) $\ln \beta$ \\
\hline 0.10 & 3207 & -10.917 & 2987. & -11.391 & 2604 & -9.894 & 2486 & -10.070 \\
\hline 0.20 & 3415 & -11.088 & 2988. & -11.045 & 3063. & -10.453 & 2829 & -10.376 \\
\hline 0.30 & 3684 & -11.375 & 3072 & -10.924 & 3398 & -10.853 & 3261 & -10.826 \\
\hline 0.40 & 4060 & -11.816 & 3191. & -10.895 & 3724 & -11.241 & $|3741|$ & -11.418 \\
\hline 0.50 & 4292 & -12.081 & 3534 & -11.266 & 3966 & -11.510 & 4083 & -11.802 \\
\hline 0.60 & 4530 & -12.350 & 3934. & -11.730 & 4265. & -11.881 & 4318 & -12.034 \\
\hline 0.70 & 4726 & -12.552 & 4154. & -11.882 & 4424. & -12.037 & 4495 & -12.193 \\
\hline 0.80 & 4791 & -12.552 & 4367. & -12.037 & 4574. & -12.183 & 4531 & -12.136 \\
\hline 0.90 & 4720 & -12.338 & 4538 & -12.128 & 4597 & -12.131 & 4682 & -12.282 \\
\hline
\end{tabular}

Se observa que los parámetros "In $\beta$ " y " $E$ " en valor absoluto, varían regularmente de forma creciente con el contenido de éster metílico en la disolución.

El hecho de que $T_{0}$ sea igual a cero, en el intervalo de temperatura estudiado, al aplicar la ecuación de Krone puede suponer que, a las temperaturas estudiadas, cada molécula vibra dentro del campo de fuerzas de sus vecinas sin desplazar a una molécula de una capa adyacente a la siguiente.

Debido a que las moléculas que forman la disolución no son esféricas, existen interacciones diferentes con sus vecinas según la dirección y, consecuentemente, estas disoluciones pueden considerarse más "sólido-líquido" que "gas-líquido". Por ello, se puede suponer que existe estructura local en dicho intervalo de temperatura, en concordancia con el modelo simplificado de "retículo" ya formulado para estas disoluciones (Muñoz, 1990) y otras similares (Flores, 1974 y 1977).

\section{CONSIDERACIONES FINALES}

Dado que los mejores ajustes a la ecuación de Krone se obtienen cuando el parámetro $T_{0}$ es igual a cero, tanto dicha ecuación como la de Andrade tienen dos parámetros, y es posible compararlas, observándose que los valores de los parámetros energéticos de ambas ecuaciones presentan las mismas secuencias de variación, si bien la ecuación de Krone resulta más adecuada como se deduce de los menores valores del porcentaje de varianza no justificada por la regresión.

En consecuencia, se confirma para las disoluciones estudiadas la validez de la hipótesis de transporte de cantidad de movimiento empleada en la deducción de la ecuación de Krone. 


\section{BIBLIOGRAFÍA}

Andrade, E.N. da C. (1930).- "The viscosity of liquids".- Nature 125, 309. Andrade, E.N. da C. (1934).- "A theory of the viscosity of liquids".- Phil. Mag. 17, 497-511.

Born, M.; Green, H.S. (1947).- "A general kinetic theory of liquids I. The molecular distribution functions".- Proc. Roy. Soc. 188 A, 10-18.

Ewell, R.H.; Eyring, H. (1937).- "Theory of the viscosity of liquids as a function of temperature and pressure".- J.Chem.Phys. 5,726-736.

Eyring, H.; Ihon,M.S. (1969).- "Significant Liquids Structures".- (Ed.) John Wiley and Sons, INC, New York.

Faeber, G.L.; Kim, S.W.; Eyring, H. (1970).- "Viscous-slow and glasstransition temperature of some hydrocarbons".- J. Phys. Chem. 74, 3510- 3518.

Flores Luque, V.; Pereda Marín, J.; Cabrera Martín, J.; Gómez Herrera, C (1974).- "Estudios sobre volúmenes específicos, tensión superficial y viscosidad en las disoluciones de aceite de oliva y hexano".- Grasas y Aceites 25, 18-28.

Flores Luque, V · Gómez Herrera, C.; Galán Vallejo, M (1977)-- "Estudios físico-químicos de miscelas de aceites vegetales II. Volúmenes parciales molares, índices de refracción y viscosidad de disoluciones de ácido oléico en hexano y ciclohexano".- Grasas y Aceites 28, 77 83.

Hogemboom, D.L.; Webb, W.; Dixon, J.A. (1967).- "Viscosity of several liquid Hydrocarbons as a function of temperature, pressure and free volume".- J. Chem.Phys. 46, 2586-2598.

Kirkwood, J.G. (1968).- "Theory of Liquids".- (Ed.) Alder B.S., New York.

Krone, R.B. (1983).- "A viscosity-temperature relation for newtonian liquids".- Chem.Eng.Commun. 22, 161-180.

Mayer, J.E.; Montroll, E. (1941).- "Molecular distribution".- J. Chem.Phys.9, $2-16$

Mayer, J.E. (1947).- "Integral equations between distribution functions of molecules".- J. Chem. Phys. 15, 187-201.

Muñoz Cueto, Mํ. J.; Galán Vallejo, M.; Flores Luque, V. (1990) "Estudios físico-químicos de aceites vegetales XII. Volúmen molar, refracción molar y viscosidad de disoluciones de miristato de metilo en ciclohexano, hexano, tetracloroetileno".- Grasas y Áceites 41, 5968.

Ree, T.; Ree, T.S.; Eyring, H. (1964).- "Significant structure theory of transport phenomena".- J. Phys.Chem.68, 3262-3267.

(Recibido: Septiembre 1993) 